\title{
Orientation-position coding and invariance characteristics of pattern discrimination
}

\author{
TERRY CAELLI \\ University of Alberta, Edmonton, Alberta, Canada \\ and \\ PETER DODWELL \\ Queen's University, Kingston, Ontario, Canada
}

\begin{abstract}
In this paper, we consider how correlated information about the orientation and position of elements in an image may be used in the detection or discrimination of patterns. Our previously reported experiments (Caelli \& Dodwell, 1982; Dodwell \& Caelli, in press) demonstrated that discrimination cannot be fully predicted from either source of information on its own. Rather, as we demonstrated, the visual system is better able to recognize and discriminate images when their position and orientation codes are highly related or define images that are invariant under similarity transformations. Here we propose and evaluate mechanisms for performing such orientation and position correlations and invariance coding that allow us to predict the discriminability of patterns. Finally, these mechanisms are shown to predict successfully the ability of observers to recognize patterns when rotated-although they are not sufficient, in themselves, to explain how the actual matching processes may occur in this latter task.
\end{abstract}

The aim of this paper is to examine the role of certain pattern codes and their correlation in the processes of pattern recognition. Further to this, we are interested in the question of how such codes and the mechanisms for correlating them may operate in the recognition of patterns under transformations ("invariance coding"). We have studied two elementary pattern codes, those for position and orientation, and patterns that are exclusively defined by these parameters (see Caelli \& Dodwell, 1982).

There have been attempts to study these issues in the past. Mach (1914) proposed not only that a pattern is coded as a set of "component sensations" of line directions, but also that recognition under transformation involves the correlation of such line-element positions and orientations (see Rock, 1973, p. 81).

Another quantitative approach was proposed by Hoffman $(1966,1978)$ and further developed by Foster (1975), Foster and Mason (1978), and Caelli and Dodwell (1980). Hoffman's fundamental postulate was that the coding of orientation at various positions of the retinotopic map by the visual system, discovered by Hubel and Wiesel (1962) and others, actually provides the visual system with "vectorfield" information. That is, the visual system, on detecting specific orientation and position states (" $\phi / P$ codes"), spontaneously extracts the path

Both authors are in the Department of Psychology of their respective institutions. The postal code at the University of Alberta is T6G 2E9; the code at Queen's University is K7L 3N6. curves (interpreted as visual contours) of which the local vectors are tangential elements.

The $\phi / P$ code along such a contour would remain invariant with respect to the transformation defined by the path curve. For example, circular $\phi / \mathrm{P}$ codes are invariant under rotations. Consequently, one can determine a degree of invariance for a pattern in terms of the relationship between its $\phi / P$ codes and some given transformation. Gibson (1950), Hoffman (1966), and Johannson (1978) have proposed that the number of such perceptually registered transformations may be quite small. For example, elements of the similarity group of transformations (rotations, translations, and dilations) that are involved in size and shape constancy would seem fundamental (so producing only a few basic "flow fields"; see Gibson, 1950).

Other attempts to relate perceptual stability under transformations to coding mechanisms have been made by Luneberg (1950), Dodwell (1970), Schwartz (1977), and Cavanagh (1978). These authors, while working from quite distinct premises and points of view, all share the notion that the mapping of the retinal image into its cortical representation is conformal. (Conformal maps preserve the local property of the angle of intersection of contours, but otherwise allow considerable freedom in the assignment of directions and positions between elements). The common limitation of these approaches is that they do not yield quantitative predictions of the extent to which images are discriminable. Although one may 
conclude from such ideas that certain patterns will be more invariant or more discriminable than others, they do not provide an explicit and quantitative formulation for how the local coding mechanisms actually affect discrimination or recognition, particularly under transformations. This paper is concerned with an initial attempt to develop such a quantitative formulation with respect to a restricted set of images.

Like Mach (1914) and the above authors, we assume that the observer, at some cortical level, generates an image code consisting of rules that associate specific orientations with image position components. These are the orientation/position rules we call $\phi / P$ codes (similar to the "primal sketch" of Marr, 1976). A class of images in which such $\phi / P$ codes give a sufficient characterization is that of "vectorpatterns," as illustrated in Figure 1. Although they are in an obvious sense quite simple, vectorpatterns can embody powerful global or "field" effects, their local elements being at the same time sufficiently well defined to permit a number of "local" manipulations to be performed on them (see Caelli \& Dodwell, 1982, for the details of how vectorpatterns are generated and manipulated).

In a recent series of experiments (Caelli \& Dodwell, 1982), we observed thresholds for discrimination between pairs of vectorpatterns as a function of orientation and position perturbations in their local elements. A number of different basic images (i.e., with different codes) were studied. The results are summarized in Figure 2 in terms of the $75 \%$ threshold values for discrimination as perturbation increased, using the sorts of orientation perturbations illustrated in Figure $1 \mathrm{~b}$. Of particular interest is the fact that orientation sensitivity is determined by the correla-

(a)

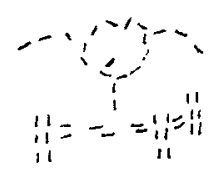

(b)
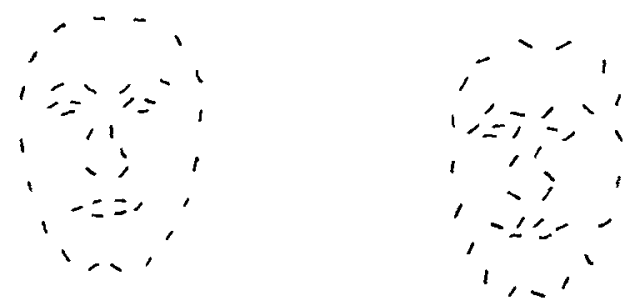

Figure 1. (a) Vectorpatterns: Images defined by orientation ( $\phi$ ) and position (P) codes $(\phi / P)$, where both can be independently manipulated. (b) Examples of original (left) and orientation perturbed (right: random perturbation of up to \pm 45 deg,) images studied by the authors (Caelli \& Dodwell, 1982).

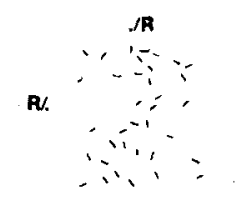

38

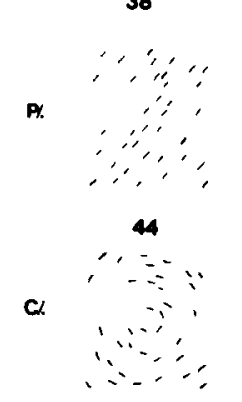

38

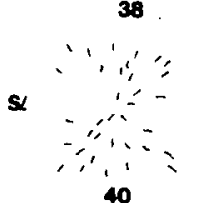

40

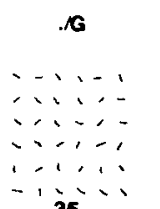

' 35
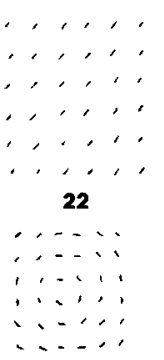

18

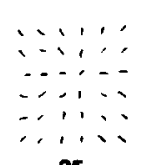

25

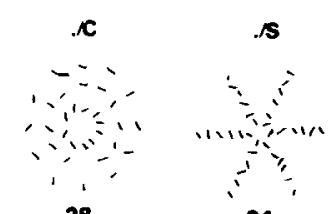

24
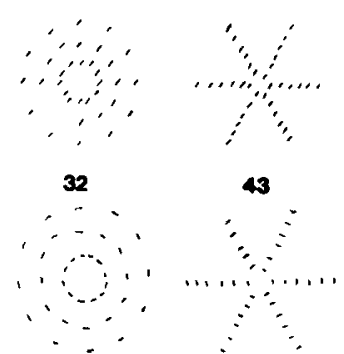

20

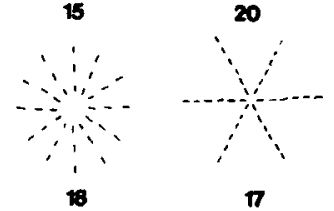

7

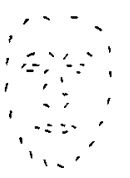

25

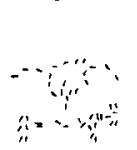

20

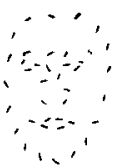

2.

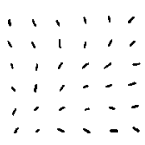

30

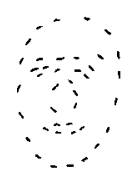

25

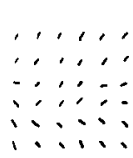

40
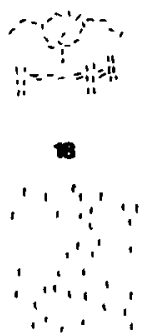

1.
Figure 2. Orientation perturbations ( $\pm \theta$ deg: bottom of each figure) yielding $75 \%$ discrimination responses from the original vectorpatterns shown. $\phi / P$ represents orientation/position codes: R, P, C, S, G-random, parallel, circular, star, grid, respectively (from Caelli \& Dodwell, 1982).

tions between position and orientation codes. When the codes are "congruent," in a sense to be discussed below, sensitivity to element perturbation is particularly high.

In a second set of experiments (Dodwell \& Caelli, in press), we studied the discrimination of vectorpatterns under rotation. That is, a basic image was displayed with a rotated and orientation-perturbed version. The task was to judge whether the rotated version was identical to the original or not. A sample of the results is shown in Figure 3. Again, it is clear that there are strong global effects (pattern-specific) on both orientation sensitivity and recognition under rotation.

Although something similar is implicit in the data of Rock (1973), we have demonstrated more formally 


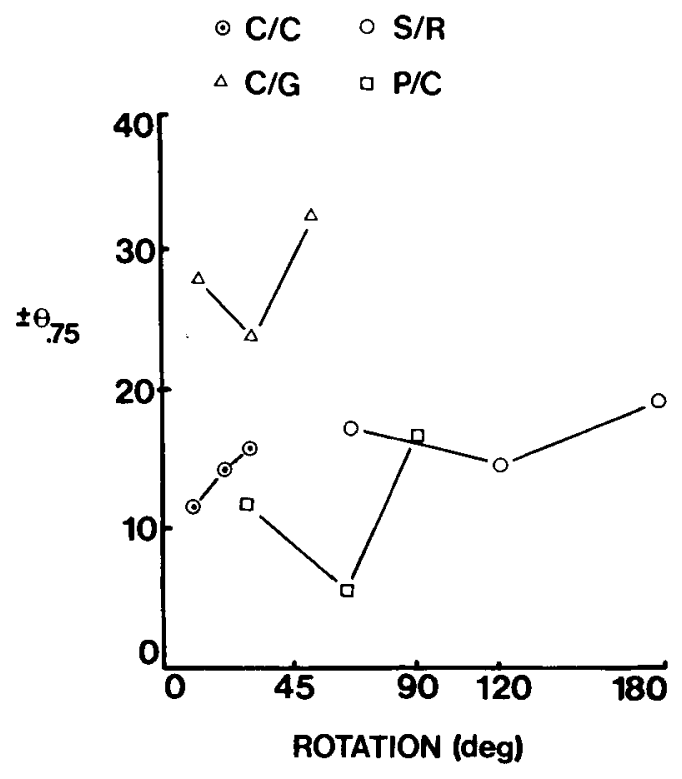

Figure 3. Orientation perturbations $( \pm \theta .75)$ yielding $75 \%$ discrimination responses for four vectorpatterns as a function of rotation angle. Rotation angles for each vectorpattern corresponded to $1 / 6,1 / 3$, or $1 / 2$ of the repetition period (from Dodwell \& Caelli, in press). $\phi / P$ denotes the specific orientation/position code (see Figure 2).

and explicitly that vectorpattern discrimination (measured by local $\phi / \mathrm{P}$ sensitivities in our paradigm) improves as the orientation and position states approach those of the path curves of similarity transformations (rotations, translations, and dilations). That is, the discrimination of perturbations in a vectorpattern is most acute when the $\phi$ and $P$ codes approach the circular, star, or grid configurations. Similarly, the data of Figure 3 show that the recognition of an object under rotations improves (in this case almost by definition) as the stimulus array approaches rotation invariance (except for the $\mathrm{P} / \mathrm{C}$ case in Figure 3, the $\mathrm{C} / \mathrm{C}$ configuration resulted in highest sensitivities or lowest orientation thresholds for discrimination).

From these results, we propose that at least two different components contribute to performance in pattern discrimination and recognition: one is related to the orientation/position correlation process ("coherence'), and another to the apparent sensitivity of specific $\phi / P$ configurations that are invariant under similarity transformations ("invariance"). In the following section, we develop a formal representation for the two processes.

\section{TWO-COMPONENTS OF VECTORPATTERN DISCRIMINATION: COHERENCE AND INVARIANCE}

\section{Coherence}

As already stated, we have found that independent considerations of the vectorpattern $\phi$ - and P-code differences are not adequate to explain vectorpattern discrimination, and that it is the relations between $\phi$ and $\mathbf{P}$ codes that best exemplify the organizational features that have perceptual salience. We now postulate a general property called coherence, which is derived from considerations about how the $\phi$ and $P$ codes may be organized perceptually in any pattern.

We assume that the visual system can arrive at a vectorpattern representation of any visual scene (within obvious limits), that is, at a set of $\phi$ and $P$ codes for any input image. We further propose that a second type of orientation information is detected from the spatial densities of elements about each position ("position-derived" orientation code), giving two orientation codes at each position. Our general idea is that when these two sources of orientation information concur, we have a pattern with high coherence. When they do not, the pattern has low coherence.

The principle is illustrated in Figure 4. Figure 4a shows a position field with strong orientational organization. This organization is enhanced when the local elements are aligned with the "positional" field (Figure 4b) or indeed when they are perpendicular to it (Figure 4c). Both yield high coherence, but $4 \mathrm{~b}$ does so more than $4 c$. When the two are totally unrelatedin Figure 4d the line elements have random orientations-coherence breaks down. Clearly, the relevant factors are: interelement distances, and the rules that constrain them; a process that assesses proximities and assigns weights to them (a filtering operationsee Caelli, 1981); and a process that relates these to the local orientation codes.

The concept of "position-derived" orientation codes $\left(\phi_{p}\right)$ follows naturally from the two elementary properties of large numbers of homogeneous cortical units: (1) spatial summation, and (2) a filter profile
A

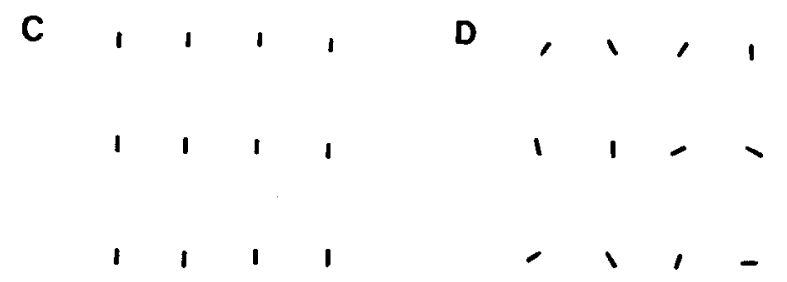

Figure 4. Degrees of vectorpatterns coherence: correlation between position and orientation codes. (a) High orientation specific position field, (b) high coherence, (c) the same, but orthogonal, (d) low coherence. 
(weighting function). With a relatively broadly tuned filter, responses are determined at each position of the vectorpattern by the sum of associated interposition distance and angle statistics (local dipole statistics; see Caelli, 1981, p. 116), weighted by the assumed perceptive field sensitivity function (Figure 5). Spatial summation of this sort, which extracts a generalized orientation response, has been discussed in detail by Caelli, Preston, and Howell (1978). The strong, local, orientation code $\phi$ is, of course, generated by detectors of the high-contrast line elements at each position in the vectorpattern. The form of the two codes is illustrated in Figure 5.

In order to simulate responses in a way consistent with known psychophysical and electrophysiological properties (e.g., see Daugman, 1980), we assume that: (1) only a finite number of orientation detectors (PFs) are operating at each position'; (2) the broadly tuned PFs which would process such positionderived orientation information (Figure 5) have a Gaussian filter profile (with decay parameter $\alpha$ ), where each finite orientation state accumulates information according to the Gaussian weighting function; (3) the "preferred" orientation ( $\phi_{\mathrm{p}}$ code) at a given position corresponds to the peak orientation response (in 2); in cases where the peak response is not unique each peak orientation is analyzed separately (Figure 5). This process can be equally well represented in the two-dimensional spatial frequency domain where the direct and "indirect" orientation codes are defined by the outputs of two channels with broad tuning in the former case and narrower orientation and frequency tuning in the latter.

Given these assumptions, we can specify quantitatively the two orientation codes at each position in the vectorpattern. If they are in close agreement at each position, we say the vectorpattern has high coherence (see above); the "coefficient of coherence" $\left[\mathrm{C}_{\mathrm{r}}(\alpha)\right]$ is defined as the correlation between the two orientation codes measured over all image positions. Here $\mathrm{C}_{\mathrm{r}}$ must be a function of the Gaussian filter profile. As $C_{r}$ approaches \pm 1 , the orientations vary together ( -1 indexing orthogonality; see Figure 4). $C_{r}(\alpha)$ can be regarded as a unitary measure of general pattern structure for the vectorpattern. For densely drawn images and simple continuous line figures, there is generally high coherence except for cases of line intersections, proximate lines, edges, etc. Appendix 1 gives a formal derivation of $C_{r}(\alpha)$. It is a measure that indexes how the visual system crosscorrelates the outputs of different sorts of spatial filters. We further propose, from the results of our previous experiments, that the correlation process may control sensitivity profiles for given orientation and position-detecting mechanisms. $\mathrm{C}_{\mathrm{r}}(\alpha)$ thus renders more precise the concept of correlation, or "congruence" between codes that we used in earlier work.

However, these considerations are not adequate to explain the results that images are not equally recognized or discriminated under similarity transformations, particularly rotations. For example, coherence, as defined above, is invariant under rotations. Con-

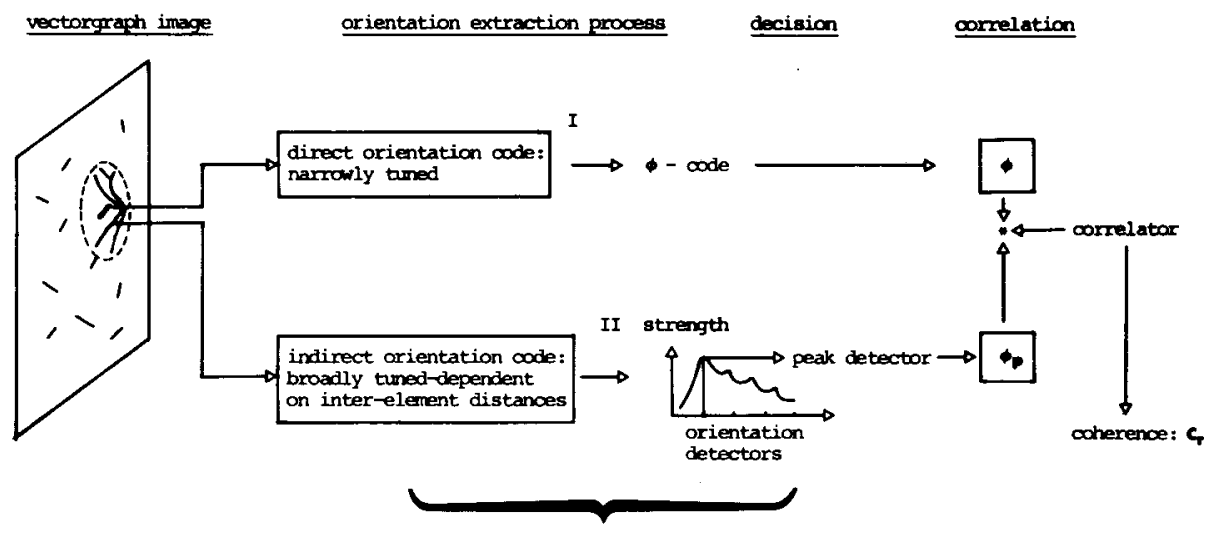

Ceneral Peroptive field profiles for $\{I$, II $\}$

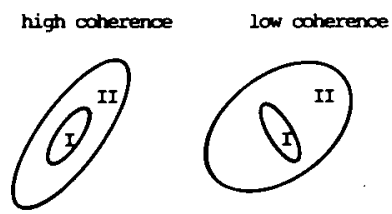

Figure 5. Vectorpattern coherence $\left(C_{r}\right)$ defined by the correlation between orientation code $(\phi)$ and position-derived orientation coding $\left(\phi_{p}\right)$ at each position. 
sequently, another type of process is required which. indexes the degree to which such vectorpatterns are invariant.

\section{Image Invariance}

Some very elementary relations hold between the $\phi$ and $P$ codes as an image undergoes a similarity transformation. Consider one such vectorpattern element as shown in Figure 6 centered at position $x_{i}$ with respect to a given origin 0 . For the orientation code to be invariant under rotations, the orientation $\phi_{i}$ at position $x_{j}$ must correspond to the angle of a tangent to a circle through $x_{i}$ centered at 0 . Further, a rotation of $x_{i}$ through angle $\eta$ about 0 must change angle $\phi_{i}$ to $\phi_{i}+\eta$. Expanding (or dilation-invariant) fields are clearly orthogonal to rotation-invariant fields. For translations, $\phi_{i}$ would be constant and independent of the position $x_{1}$. Consequently, for any position $x_{i}$, an orientation code $\phi_{i}$ must be of the linear form ( $\mathrm{a}, \mathrm{b}$ constants):

$$
\phi_{\mathrm{i}}=\mathrm{a} \eta_{\mathrm{i}}+\mathrm{b}
$$

for the vectorpattern to be invariant under similarity transformations (Appendix 2 gives the full derivation).

It follows from this simple rule that the correlation between all position displacement angles (Figure 6) and the corresponding orientation value results in a measure of transformation invariance, which we term I. When the secondary source of orientation information is employed (i.e., position-derived orientation, as defined for coherence), a different invariance measure can be determined. This we define by $I_{p}(\alpha)$, because it, like coherence, is dependent on the filter or weighting-function limits (Appendix 2).

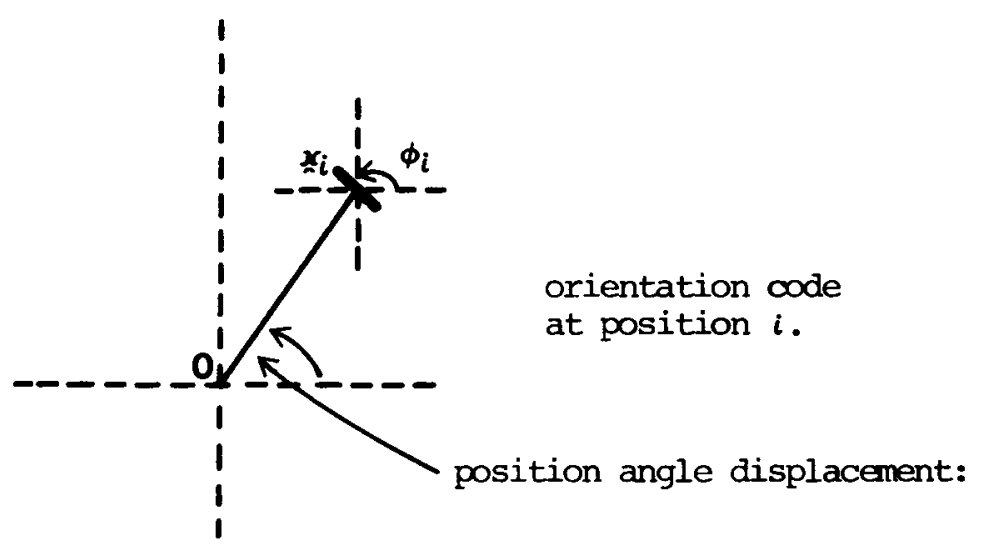

transformation center

(rotations, dilations)
Relations Between $C_{R}, I$, and $I_{p}$

Clearly, $C_{r}, I$, and $I_{p}$ capture different aspects of the vectorpattern's structure and are somewhat independent, although $C_{r}$ places some limitations on possible relationships between $I$ and $I_{p}$. These measures may assume quite different values for the same images. Two obvious examples illustrate the point: (1) With a random position code, $C_{r}$ will generally be low except for the highly improbable case in which the orientation code very closely matches the filter profiles. On the other hand, I could be any value; as already noted, a circular orientation code on the random-position field would result in $I=1.0$, for example. However, for any random-position code, $I_{p}$ will generally be low, since the likelihood that the position-derived orientations will correlate with those for the similarity transformations is very low. (2) For a random-orientation code, I will necessarily be close to zero, whereas $C_{r}$ and $I_{p}$ will assume values that depend on the position code (e.g., a vectorpattern with a star position code can have moderately high coherence, even when the orientation code is random; see Figure 2). In the following sections, we shall analyze some previously reported results in terms of coherence and invariance and also develop their characteristics further in specific applications.

\section{SIMULATION OF VECTORPATTERN RESULTS}

Although we know that vectorpattern discrimination within the orientation perturbation paradigm can be partially accounted for in terms of variance of the orientation code (Caelli \& Dodwell, 1982, Figure 7), we have shown that a major discrimination component is based on the relationship between ori-

Figure 6. Invariance analysis, demonstrating the linear relationship between position orientation $\left(\eta_{i}\right)$ change and orientation changes of a vector $(\phi)$ defined posi$\operatorname{tion} \mathbf{x}_{\mathbf{i}}$. 
entation and position codes. For example, the vectorpatterns in each of the first four rows of Figure 2 have identical orientation codes and yet the discrimination thresholds vary within each row. This cannot be predicted by either orientation or position distributions alone (Caelli \& Dodwell, 1982).

Coherence analysis requires one to set the appropriate values for orientation and distance sensitivity (Appendix 2), the latter in terms of a half-amplitude value. The average coherence coefficient $\left[C_{r}(\alpha)\right]$ for a number of such half-amplitude values was calculated over the vectorpatterns shown in Figure 2, excluding the four parallel orientation cases, which have no orientation variance, and are therefore inappropriate. This function is shown in Figure 7a, yielding greater coherence values in the $1 \leqslant \alpha \leqslant 1.5 \mathrm{deg}$ visual angle range (the overall visual angle of each vectorpattern being 4 deg in our experiments).

Employing $\alpha=1 \mathrm{deg}$ and 18 orientation detectors of 10-deg widths, Figure $7 \mathrm{~b}$ shows the $\mathrm{C}_{\mathrm{r}}(\alpha)$ values over the 12 vectorpatterns. Polynomial regression was then used to compare observed vectorpattern discrimination sensitivity ${ }^{2}$ (DS) with the coherence measures. A significant quadratic component was found $[F(1,9)=1.45, p>.10]$ with a multiple regression coefficient of 0.89 .

Also shown in Figure 7c are data for the face, scene, and special function vectorpatterns of Figure 2 (bottom row). Finally the four parallel orientation cases are shown. Although $\mathrm{C}_{\mathrm{r}}(\alpha)$ is not defined for images with zero orientation variance, we added a random \pm 1 -deg jitter to each orientation to produce the data points. $C_{\mathrm{r}}(\alpha)$ is a bad predictor of sensitivity when the orientations are all nearly parallel, which is not surprising, because we have employed a correlation rather than a direct difference measure. The problem with the latter is that, in general, it does not adequately measure the covariation of the two codes.

Figure 8 shows the relationship between positionderived invariance $\left(I_{p}\right.$ for $\alpha=1$ deg of visual angle, as before) and discrimination sensitivity for the 12 vectorpatterns analyzed in Figure $7 \mathrm{~b}$. Excluding the $\mathrm{R} / \mathrm{C}$ and $\mathrm{R} / \mathrm{S}$ cases (random orientation code cases) and the parallel orientation cases, a clear relationship was observed between $I_{p}$ and orientation discrimination sensitivity (multiple correlation coefficient of $\mathrm{R}=\mathbf{0 . 8 9}$, Figure 8). For such patterns, the I statistic, based solely on the orientation code, is irrelevant to the prediction of discrimination sensitivity differences, because parallel, circular, and star cases all have I values of unity, the random orientation code having approximately zero I values, by definition as we explained above.

The finding that the random-orientation code significantly affects discrimination illustrates the twocomponents involved in such pattern discriminations. However, a multiple regression of both $C_{\mathrm{r}}(1.0)$ and $I_{p}(1.0)$ components against the discriminability of these same 12 vectorpatterns resulted in a multiple $R$ of 0.92 , explaining $84 \%$ of the variance, with both contributing approximately equally $\left[C_{r}(1.0)\right.$ coefficient of $0.19, I_{p}(1.0)$ coefficient of 0.25 ].

These simulations demonstrate that both coherence and invariance, as we have defined them, capture important-and somewhat different-aspects of pattern discrimination, as measured in our paradigm.
A

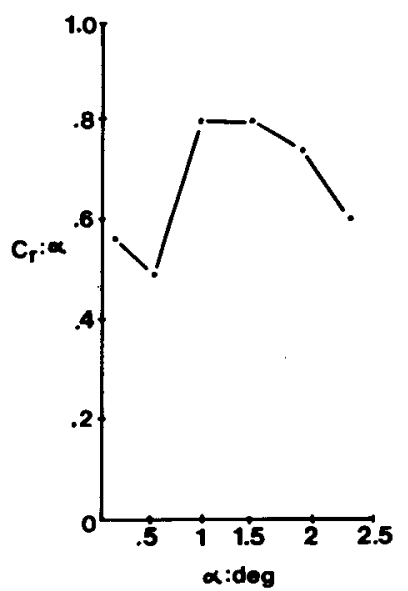

B

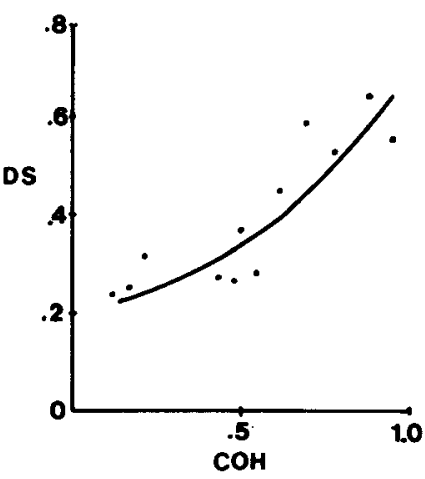

C

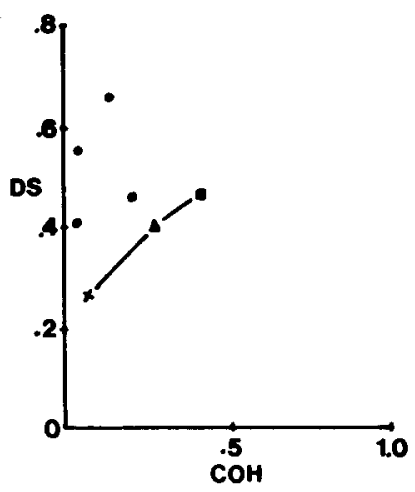

Figure 7. (a) Average coherence values $\left[C_{r}(\alpha)\right]$ over the 12 vectorpatterns in Figure 3 (rows 1, 3, 4) for a range of Gaussian filters of $1 / 2$ amplitudes (1/2 amp: $\alpha$ ) in degrees of visual angle. Vectorpattern angle was 4 deg. (b) Relationship between vectorpattern coherence $(1 / 2$ amplitude $=1 \mathrm{deg})$ and orientation sensitivity $(10 /$ orientation perturbation resulting In $\mathbf{7 5 \%}$ discrimination) for the 12 vectorpatterns in (a) above: DS. Significant quadratic regression function is shown $(R=0.89)$. (c) DS values (6) for row 2 of Figure 2 (parallel code) with induced perturbations of \pm 1 deg about the fixed 45 -deg angle. $\square, A, x$ correspond to the scene, face, and SF1 images of Figure 3 (row 6 , col. 1; row 5, col. 1; row 5, col. 2 , respectively; $1 / 2$ amplitude= 1 deg). 


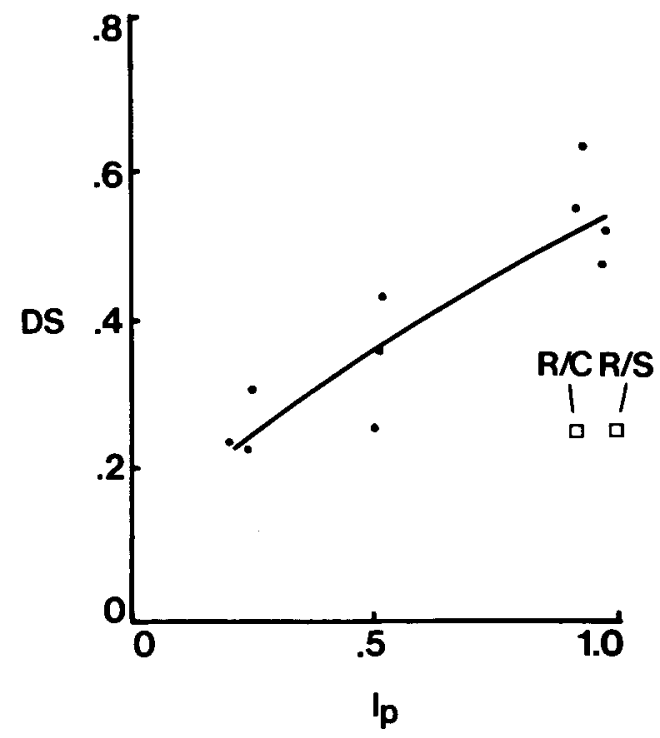

Figure 8. Relationship between orientation sensitivity (DS: 10/ orientation perturbation resulting in $75 \%$ discrimination) and position-derived invariance (I: $\alpha=1 \mathrm{deg}$ for $1 / 2$ amplitude of Gaussian filter) for the 12 vectorpatterns analyzed in Figure 7. Square points correspond to patterns with random orientation codes on structured fields. The significant quadratic regression $(R=0.89)$ is shown-excluding $R / C$ and $R / S$ cases.

But this does not imply that simple position and orientation statistics as such, play no role. On the contrary, we showed earlier (Caelli \& Dodwell, 1982) that vectorpattern discrimination does tend to be correlated with certain properties of the position and/or orientation codes themselves. Nevertheless, it is clear that, with the properties we have examined, sensitivity increases with the postulated invariance characteristics $\left(I, I_{p}\right)$ and covariation $\left(C_{r}\right)$ of both orientation codes. This does not exclude the possibility that other types of correlation processes might be involved in discriminatory behavior. However, the mechanisms we postulate are derived simply from known detector profiles of the vertebrate visual system, and a straightforward assumption of increased sensitivity to codes that remain invariant under some natural transformations of images.

\section{RECOGNITION UNDER TRANSFORMATIONS}

The invariance component of image organization, indexed by the $I$ and $I_{p}$ statistics, clearly addresses the problem of image recognition under transformation. A vectorpattern's $\phi / P$ code will remain more or less constant under similarity transformations as a function of I's and $I_{p}$ 's proximity to unity. This, in turn, implies that the perceptual identity of the image under transformation is predictable from these measures.

The idea that recognition under transformation is a function of the $\phi / P$ codes was investigated by
Dodwell and Caelli (in press) in experiments on vectorpattern discrimination under rotations. The observer's task was to discriminate between a basic image and an orientation-perturbed rotated version of it. A discrimination paradigm was used in order to avoid the uncontrolled part-response problems associated with recognition paradigms, and also to observe whether the orientation perturbation thresholds would be directly related to those observed in the unrotated cases and to the $I, I_{p}$ measures. In particular, it follows from this formulation that a lower orientation perturbation discrimination threshold implies better recognition under rotations. Results are shown in Figure 9 for one experiment with four different vectorpatterns (Dodwell \& Caelli, in press, Experiment 1) in which discrimination sensitivities ${ }^{2}$ were averaged over all seven rotation angles employed ( 0 to 180 deg in 30-deg steps).

Similarly, Figure 10 shows recognition scores for three representative shapes used by Rock (1973) at 45-, 90-, and 180-deg rotation states, using a recognition memory paradigm. By reducing such images to $\phi / P$ codes [all with extremely high coherence $\left(C_{r}\right)$ ], we have analyzed these data in terms of the $I_{p}$ invariance statistic (all filters defined by Equations 2-4 in Appendix 1 were set at $\alpha=1 \mathrm{deg}$ of visual angle with respect to a 4-deg total image visual angle). Again, we have not employed the I statistic in either of these cases (Figures 9 and 10), since, for the vectorpatterns used, I was unity and for the Rock shapes (Figure 10), I and $I_{p}$ are highly correlated because of the continuous nature of the contours employed.

Here (Figures 9 and 10) the relationship between discrimination sensitivity (DS) and the positionderived invariance coefficient $\left(I_{p}\right)$ is shown. As before, these results show that as $I_{p}$ increases, the

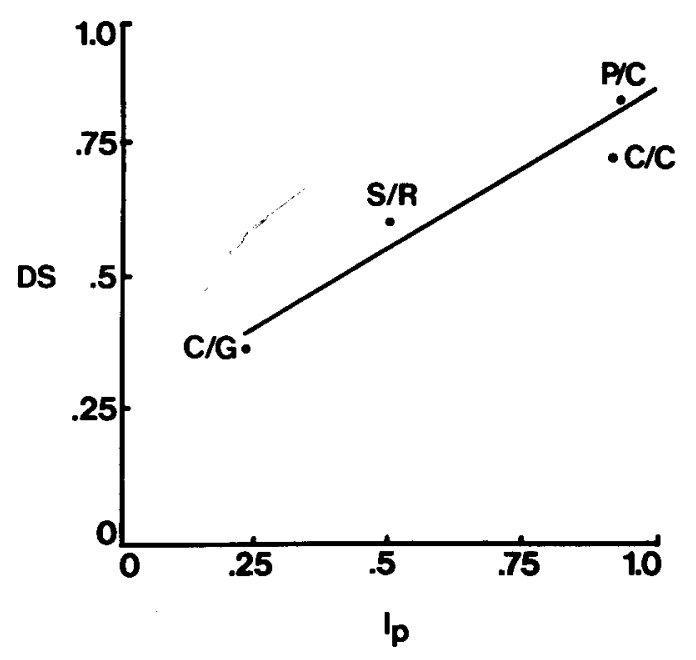

Figure 9. Relationship between position-derived invariance $\left(I_{p}\right.$ : $\alpha=1 \mathrm{deg}$ ) and orientation sensitivity (DS: 10/orientation perturbation resulting in $75 \%$ discrimination, averaging over pattern rotations (Dodwell \& Caell, in press). 


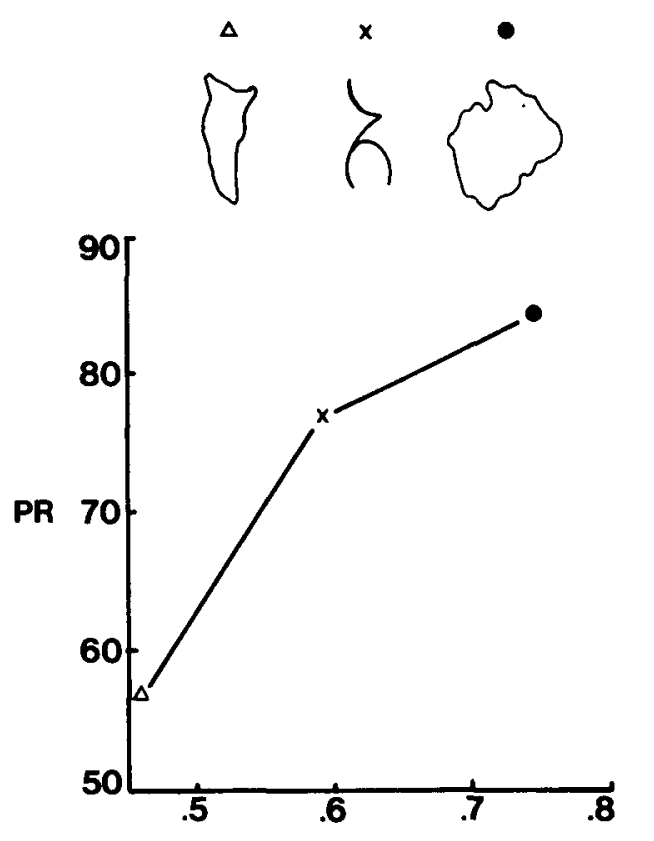

Figure 10. I values for three stimuli of Rock (1973) compared with recognition values (percentage recognition: PR) averaged over rotation angles ( $a=1$ deg: 1/2 amplitude of Gaussian Filter).

(orientation-based) discriminability improves, suggesting that the position-derived invariance property indexes aspects of orientation sensitivity in this task. Although only four vectorpatterns and three of the Rock shapes have been analyzed in detail, these images are reasonably representative. The coherence measure $\left(C_{r}\right)$ yielded no systematic relationship with discrimination sensitivity under the rotation states, as expected. This indicates that the matching process between an image and its rotated version is not simply based on the covariation of the two codes.

The invariance coefficients we have developed do not capture all the processes measured in this type of experiment. For example, they do not account for the observed strong interactions between image type and rotation state; in general, different classes of images show different patterns of recognizability under different amounts of rotation (Dodwell \& Caelli, in press). The point of the invariance coefficients was to develop a general index of the degree to which any matching process between an image and its transformed version is possible. It does not yield specific predictions for pattern rotation interactions. Also, recognition under transformation requires the postulation of a process that codes magnitude of transformation. One of the salient features of perception under transformation is that recognition both of identity and of the transformation itself generally occur. Modeling both these processes simultaneously in the context of image coding presents formidable difficulties; to the best of our knowledge, no one has ever considered this problem before.
A process is required that registers the transformation magnitude. This may be akin to the "mental rotation" studied by Shepard and his colleagues (Shepard, 1981), but in our experimental situation the process is not mental rotation as this is now conventionally understood. The reason is that mental rotation takes time, that is, judgment time is systematically related to transformation magnitude. In our experiments, there was no such relationship. In some sense, our judgments of similarity were more immediate than those involved in mental rotation (Holland \& Deluis, 1982). Nevertheless, transformation magnitude is registered in some way, and the question remains as to what the mechanism may be. Thus far, we have considered functions relating orientation and position codes within each image. If the approach is to be useful, it seems that we also need to develop measures of the correlation of codes between images.

In attempting to address this problem, three general alternatives seem possible: (1) a mechanism that either "knows" or computes the transformation and then determines the "fit" between the two $\phi / P$ codes; (2) a mechanism that "fits" the $\phi / P$ codes and then determines the best transformation; or (3) some recursive combination of (1) and (2). "Mental rotations" are of the first type and presume that the observer, either through past experience or through other sensory information, can accommodate to the transformation in order to judge similarity. The second type follows Mach (1914), where the degree to which an object and its transformed version are identified is a function of the similarity between orientation components at each position in the visual field.

To the extent that coherence $\left(C_{r}\right)$ does not predict recognition under transformation, we may suppose that a mechanism of type 1 is involved. This conclusion was also reached, on somewhat different grounds, by Dodwell and Caelli (in press) in interpreting their psychophysical measures. $I$ and $I_{p}$ measure the degree to which $\phi / P$ codes remain invariant under similarity transformations, and the fact that they do predict performance in discriminationunder-rotation tasks (Figures 9 and 10) also supports the idea of a type 1 mechanism. Finding the appropriate transformation is only half of the story, however. There must be a form of comparison between images, a "proximity match" to establish the similarity of the images following transformation.

\section{CONCLUSIONS}

In conclusion, we reiterate our claim to have demonstrated that at least two forms of pattern processing are involved in the recognition of the vectorpatterns and their transformations. Presumably the same is true of other forms of two-dimensional spatial patterns. Our measures of coherence and in- 
variance are, in a sense, one attempt to bridge the gap between the two. Coherence is very much a "local" correlation phenomenon, readily interpreted in terms of what we know of vertebrate receptive field properties. Invariance, on the other hand, and especially I, the orientation-specific invariance, is a direct measure of image invariance under similarity transformations. $I_{p}$, the position-derived measure of invariance, is intermediate between the two, and turns out to be the more useful and discriminating measure of the invariance properties of images that do not embody the similarity transformation fields (rules) themselves.

How it is that an observer can both recognize the identity (or similarity) of two patterns, on the one hand, and also "see" the transformation to which they have been subjected, on the other? Our suggested resolution is made in very general terms. Perhaps the identity-under-transformation match is attained by some form of local $\phi / P$ comparison mechanism, the recognition (and hence operation) of the global transformation itself by the application of transformation rules. Our argument at the end of the previous section suggests that a subjective image transformation is at least concurrent with local proximity matching. The similarity transformations have a special relevance to object constancy, and it is quite possible that the systems for computing them are built into the very anatomical structure of the visual cortex, as suggested originally by Hoffman (1966) and subsequently proposed, from a somewhat different perspective, by Schwartz (1977). What we have attempted in this paper is to put certain processes involved in pattern recognition/discrimination into a quantitative and coding perspective. The measures we have derived to test these processing characteristics restrict somewhat both the class of objects and behaviors we can investigate, although we hope that the principles themselves may turn out to be general.

\section{REFERENCES}

Caelli, T. M. (1981). Visual perception: Theory and practice. Oxford: Pergamon Press.

Caflei, T. M. (1983). Energy processing and coding factors in texture discrimination and image processing. Perception \& Psychophysics, 34, 349-355.

Caelli, T. M., \& Dodwell, P. C. (1980). On the contours of apparent motion: A new perspective on visual space-time. Biological Cybernetics, 39, 27-35.

Caelli, T. M., \& Dodwell, P. C. (1982). The discrimination of structure in vectorgraphs: Local and global effects. Perception \& Psychophysics, 32, 314-326.

Caelli, T. M., Preston, G., \& Howell, E. (1978). Implications of spatial summation models for processes of contour extraction: A geometric perspective. Vision Research, 18, 724-734.

Cavanagh, P. (1978). Size and position invariance in the visual system. Perception, 7, 167-177.

Daugman, J. (1980). Two-dimensional spectral analysis of cortical receptive field properties. Vision Research, 20, 847-856.
Dodwell, P. C. (1970). Visual pattern recognition. New York: Holt, Rinehart and Winston.

Dodwell, P. C., \& CAelli, T. M. (in press). Recognition of vectorpatterns' under transformations: Local and global determinants. Quarterly Journal of Experimental Psychology.

Fosten, D. (1975). Visual apparent motion and some preferred paths in the rotation group SO(3). Biological Cybernetics, 18, 81-89.

Foster, D., \& Mason, R. (1978). Transformation and relationalstructure schema for visual pattern recognition. Biological Cybernetics, 32, 85-93.

GiBson, J. (1950). The perception of the visual world. Boston: Houghton Mifflin.

Hoffman, W. C. (1966). The Lie algebra of visual perception. Journal of Mathematical Psychology, 3, 65-98.

HoFFMAN, W. C. (1978). The Lie transformation group approach to visual neuropsychology. In E. Leewenberg \& H. Buffart (Eds.), Formal theories of visual perception (pp. 27-66). New York: Wiley.

Holland, V. D., \& Deluis, J. D. (1982). Rotational invariance in visual pattern recognition by pigeons and humans. Science, 218, 804-806.

Hubel, D., \& Wiesel, T. (1962). Receptive fields, binocular interaction and functional architecture in the cat's visual cortex. Journal of Physiology (London), 160, 106-154.

Johannson, G. (1978). About the geometry underlying spontaneous visual decoding of the optical message. In $E$. Leewenberg \& R. Buffart (Eds.), Formal theories of visual perception (pp. 265-276). New York: Wiley.

LUNEBerg, R. (1950). The metric of binocular visual space. Journal of the Optical Society of America, 40, 627-642.

MaCH, E. (1914). The analysis of sensations. Chicago: Open Court.

Marr, D. (1976). Early processing of visual information. Proceedings of the Royal Society (London), 275, 483-519.

Rock, I. (1973). Orientation and form. New York: Academic Press.

Schwartz, E. (1977). Spatial mapping in the primate sensory projection: Analytic structure and relevance to perception. Biological Cybernetics, 25, 181-194.

Shepard, R. (1981). Psychophysical complementary. In $\mathbf{M}$. Kubovy \& J. R. Pomeraut (Eds.), Perceptual organization. Hillsdale, NJ: Erlbaum.

\section{NOTES}

1. In our case, we have assumed 18 detectors of 10-deg tuning width (Figure 6), consistent with recent results on the inability of observers to discriminate orientation information as briefly exposed textures of greater resolution than \pm 5 deg (Caelli, 1983).

2. $\mathrm{DS}=10 . / \theta .75, \theta_{.75}$ being the orientation perturbation threshold: the $75 \%$ discrimination point on the psychometric function.

\section{APPENDIX 1}

\section{Formal Derivation of Coherence, $\mathbf{C}_{\mathrm{r}}(\boldsymbol{a})$}

The position derived orientation $\phi_{p}(i)$ at position $X_{i}$ is formally obtained as follows. For homogeneous application of the Gaussian filter and orientation distribution, we have the net response of orientation detector $k: \phi_{p}(k / i)$ determined by:

$$
\phi_{p}(k / i)=\frac{1}{\sigma \sqrt{2 \pi}} \int_{-\infty}^{\infty} \int_{\phi_{1}}^{\phi_{u}} \delta(r) e^{-1 / 2(r / a)^{2}} r d \phi d r,
$$

where $\mathrm{r}$ corresponds to visual separation; $o$ the standard de- 
viation: $\phi_{\ell}, \phi_{1}$ to the angular bounds defining the tuning width for orientation detector $k ; \delta(r)$ is the Dirac delta function, which determines the filter point spread function (Caelli, 1981, p. 128). Thus Equation 1 reduces to:

$$
\phi_{p}(k / i)=\frac{1}{\sigma \sqrt{2 \pi}} \sum_{j=1}^{n^{*}} e^{-1 / 2\left(r_{i j} / \sigma\right)^{2}},
$$

where $n *$ corresponds to the number of positions $\left(x_{j}\right)$ that lie in the segment determined by $\phi_{\ell}, \phi_{u}$. We then assume the threshold function:

$$
T\left(\phi_{p}\left(k^{*}, o / i\right)\right)=\max _{k}\left\{\phi_{p}(k, o / i)\right\}
$$

which associates the orientation $k^{*}$ to position $x_{i}$, since it corresponds to the peak amplitude over all orientations. That is:

$$
\phi_{\mathrm{p}}(\sigma: \mathrm{i})=\mathrm{T}\left(\phi_{\mathrm{p}}(\mathrm{k}, \sigma / \mathrm{i})\right) .
$$

We then define $C_{r}$ as the correlation between $\phi_{p}(\alpha: i)$ and $\phi_{\mathrm{j}} / \mathbf{X}_{\mathrm{i}}$, the actual orientation of the line element at this position. $C_{r}$ is a function of the Gaussian width (o), which is more realistically determined by the half-amplitude value $\alpha$, where $\alpha=(\sigma / 2) \ln 2$. That is:

$$
C_{r}(\alpha)=\frac{\operatorname{cov}\left\{\phi_{p}(\alpha: i), \phi / x_{i}\right\}}{\sigma_{\phi_{p_{i}}} \cdot \sigma_{\phi / x_{i}}} .
$$

This $\mathrm{C}_{\mathrm{r}}(\alpha)$ is the coefficient of coherence, which corresponds to one component in vectorpattern processing (Figure 6). It is an index of feature extraction generated by the action of filters which are sensitive to intrinsic orientation and distance parameters of the image components.

\section{APPENDIX 2}

\section{Formal Derivation of Two Invariance Measures: I,I( $\alpha)$}

The second component of vectorpattern structure is concerned with how the $\phi / P$ codes are preserved under transformations, in particular the similarity transformations discussed above. We develop coefficients of pattern invariance which index the degree to which the image $\phi / P$ codes are invariant under the general linear transformations:

$$
\begin{aligned}
& x^{1}=a_{1} x+b_{1} y+c_{1} \\
& y^{1}=a_{2} x+b_{2} y+c_{2}
\end{aligned}
$$

where $a_{1} b_{2}-a_{2} b_{1} \neq 0$. For rotations, translations and dilations, Equation 6 assumes the forms: (a) Rotations

$$
\begin{aligned}
& \mathrm{x}^{1}=\left(\mathrm{x}-\mathrm{x}_{0}\right) \cos \psi+\left(\mathrm{y}-\mathrm{y}_{0}\right) \sin \psi+\mathrm{x}_{0} \\
& \mathrm{y}^{1}=-\left(\mathrm{x}-\mathrm{x}_{0}\right) \sin \psi+\left(\mathrm{y}-\mathrm{y}_{0}\right) \cos +\mathrm{y}_{0}
\end{aligned}
$$

about the center $\left(\mathrm{x}_{0}, \mathrm{y}_{0}\right)$.

(b) Dilations

$$
\begin{aligned}
& x^{1}=\alpha\left(x-x_{0}\right)+x_{0} \\
& y^{1}=\beta\left(y-y_{0}\right)+y_{0}
\end{aligned}
$$

with respect to the center $\left(\mathrm{x}_{0}, \mathrm{y}_{0}\right)$.

(c) Translations

$$
\begin{aligned}
& x^{1}=x+\alpha \\
& y^{1}=y+\beta
\end{aligned}
$$

Now, the direction field (invariant vector field) associated with a given position $x_{i} \equiv\left(x_{i}, y_{i}\right)$ has orientations determined by:

$$
\left\{\tan ^{-1}\left[-\left(x_{i}-x_{0}\right) /\left(y_{i}-y_{0}\right)\right], \tan ^{-1}\left[\left(y_{i}-y_{o}\right) /\left(x_{i}-x_{o}\right)\right], \beta / \alpha\right\}
$$

for rotations, dilations, and translations, respectively, as shown in Figure 6.

Rotations and dilation fields are orthogonal, so it is clear from the above and Figure $6 \mathrm{~b}$ that the regression equation:

$$
\phi_{i}=a \eta_{i}+b
$$

(where $\eta_{i}$ is the angle subtended at the origin by the element at $\mathrm{x}_{\mathrm{j}}$ ) and the associated correlation coefficient determines the degree to which a given $\phi / P$ code corresponds to the invariant paths of the transformations. In Equation $7, \phi_{i}$ corresponds to the element orientation defined at a given position $x_{i}$. We can equally correlate the position-derived orientation $\phi_{p}(i)$ as defined in Equations 2-4 with the displacement angle $\eta_{\mathrm{i}}$ to result in the correlation function:

$$
I_{p}\left(\eta, \phi_{p}: \alpha\right)=\frac{\operatorname{cov}\left\{\phi_{p}(\alpha: i), \eta_{i}\right\}}{\sigma_{\phi_{p}}(\alpha: i) \cdot \sigma_{\eta_{i}}}
$$

which we call the "position-derived" invariance of a vectorpattern.

(Manuscript received February 25, 1983; revision accepted for publication June 7,1984 .) 